\title{
Editorial
}

\section{Dynamics, Control, and Optimization with Applications 2014}

\author{
Ryan Loxton, ${ }^{1}$ Qun Lin, ${ }^{1}$ Shengjie $\mathrm{Li}^{2}{ }^{2}$ and Guanglu Zhou ${ }^{1}$ \\ ${ }^{1}$ Curtin University, Perth, WA 6102, Australia \\ ${ }^{2}$ Chongqing University, Chongqing 400044, China
}

Correspondence should be addressed to Ryan Loxton; r.loxton@curtin.edu.au

Received 11 June 2014; Accepted 11 June 2014; Published 21 July 2014

Copyright (C) 2014 Ryan Loxton et al. This is an open access article distributed under the Creative Commons Attribution License, which permits unrestricted use, distribution, and reproduction in any medium, provided the original work is properly cited.

Dynamic systems abound in virtually all areas of science and engineering, and control and optimization strategies for such systems play a crucial role in many applications, including batch chemical reactions, spacecraft control, and electronic circuit design. The aim of this special issue is to present recent advances in the areas of dynamic systems, control, and optimization and explore the interplay between them.

This special issue is the sequel to a previous special issue published last year in this journal on the same topic. The topics covered in the special issue range from fundamental mathematical theory to applications in mathematical finance and space trajectory optimization. Given this excellent mix of strong papers from a variety of different areas, we believe that this year's special issue has been as successful as, if not more than, the original special issue published in 2013.

\section{Acknowledgments}

We would like to express our sincere gratitude to all authors for their contributions to the special issue. We would also like to thank the reviewers for their generous help in reviewing the submitted manuscripts. Their efforts were instrumental to the success of this special issue.

\author{
Ryan Loxton \\ Qun Lin \\ Shengjie Li \\ Guanglu Zhou
}




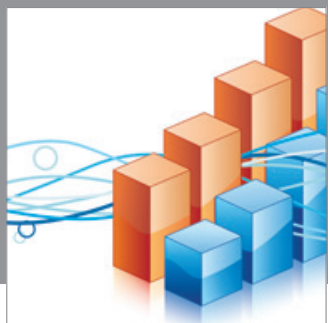

Advances in

Operations Research

mansans

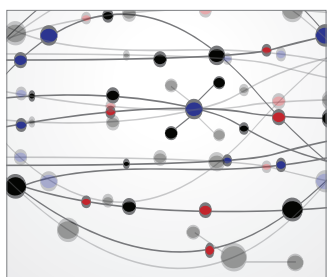

The Scientific World Journal
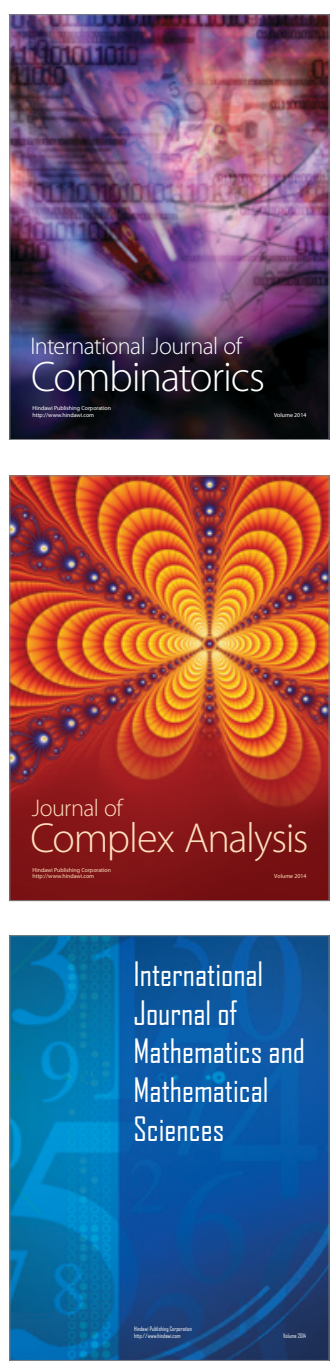
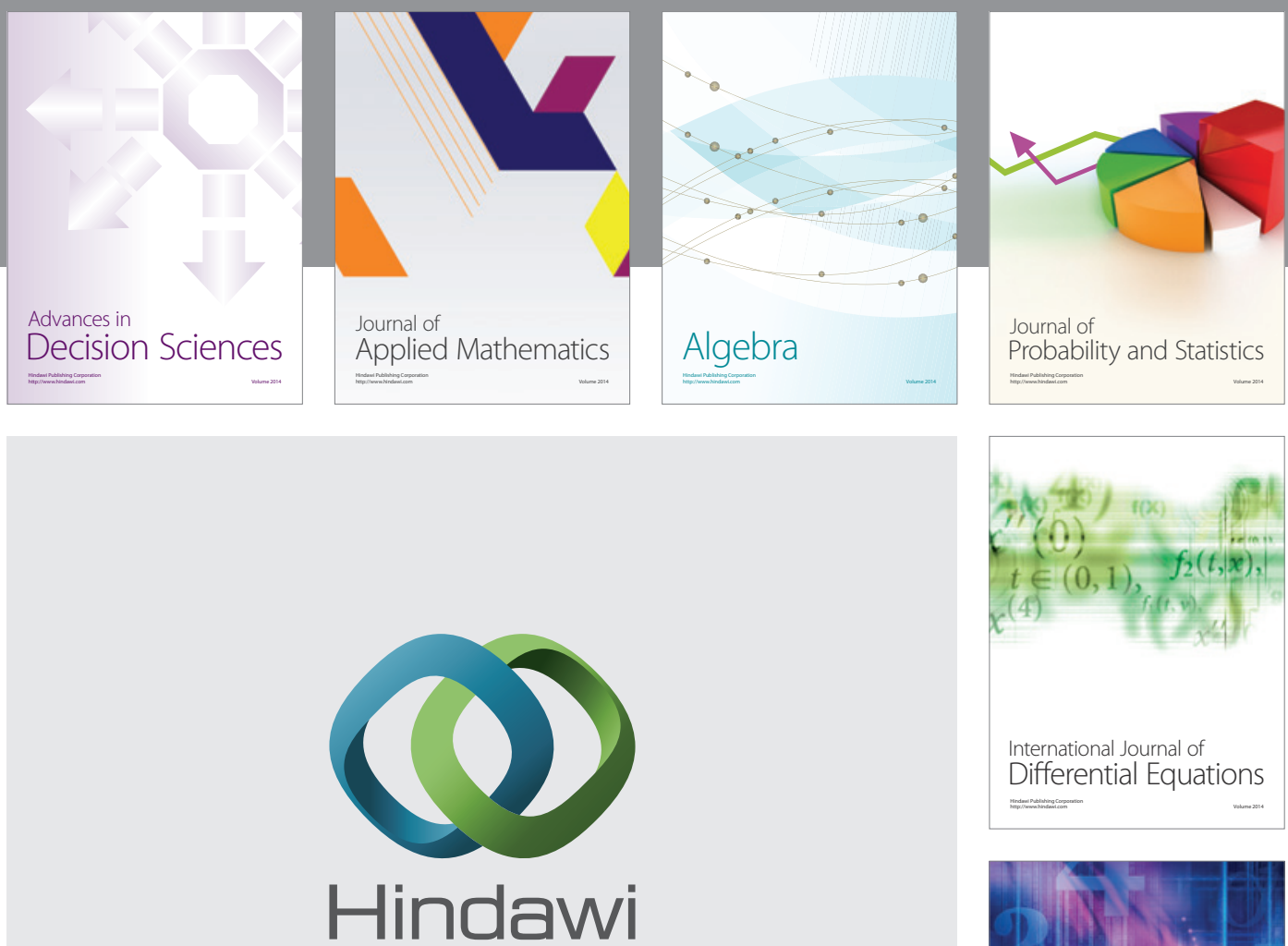

Submit your manuscripts at http://www.hindawi.com
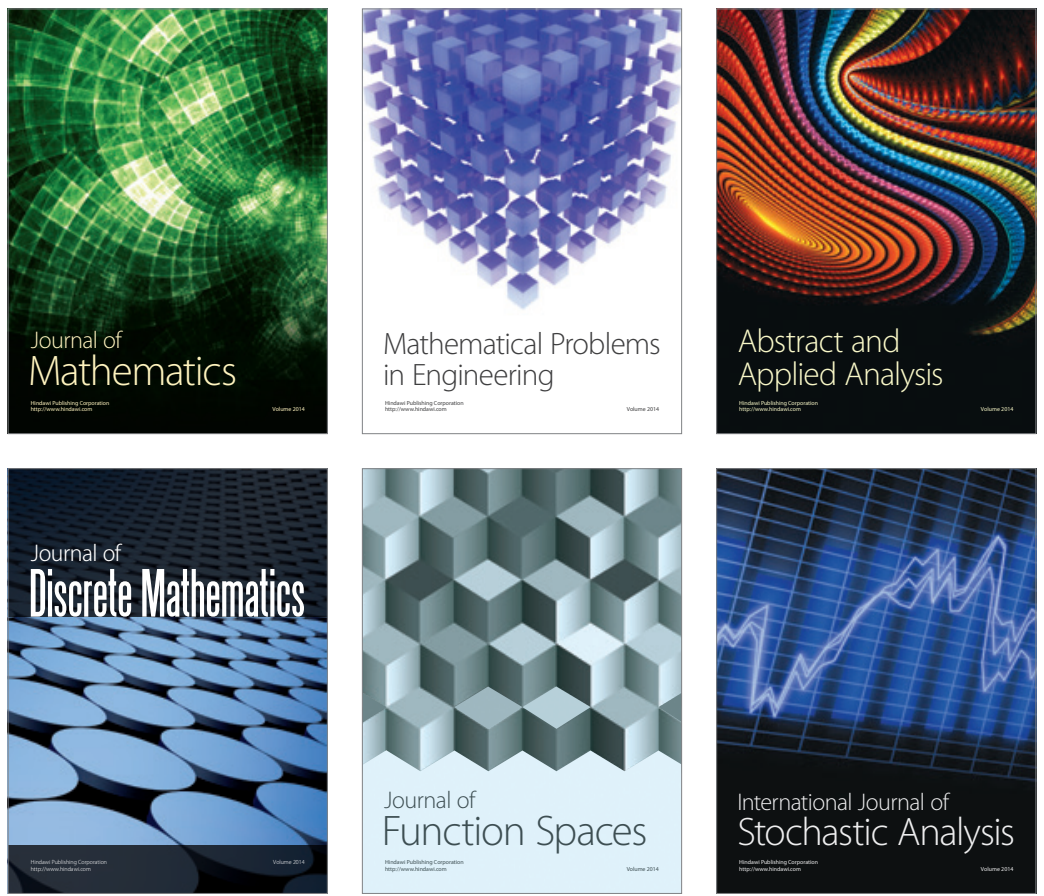

Journal of

Function Spaces

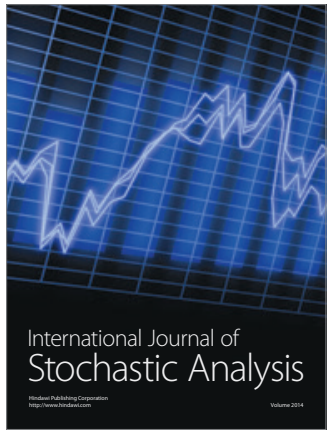

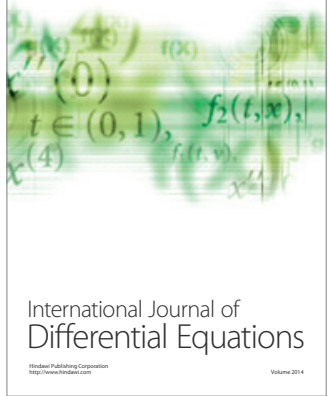
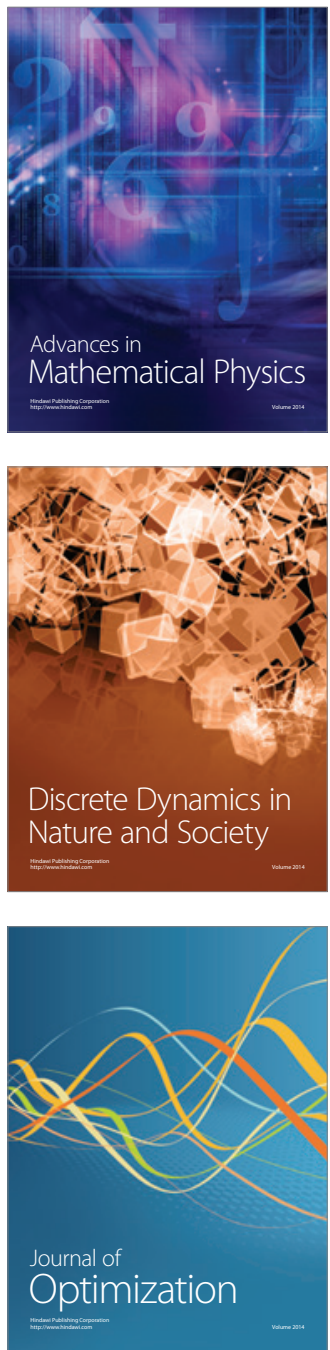